(ADHD) alone does not carry a poor prognosis unless it is in conjunction with serious functional impairment.

There remain numerous unresolved issues within the field of attentional and hyperactivity disorder research and practice and this book addresses many of them. For example, what does one do with children who meet criteria in one environment but not another? How do sub-threshold cases fit into our understanding of the stability of these disorders? One chapter highlights the uncertainties of the reliability and validity of some measures widely employed in research and practice. There is also exploration of the false assumption that behavioural inattention and inhibition should be reflected in the laboratory measurement of inattention and inhibition. Emerging neuropsychological literature increasingly highlights the misnomer of ADHD: many children with clinically diagnosed ADHD or hyperkinetic disorder do not demonstrate deficits in certain aspects of attentional processing. Rather, a deficit in state regulation has been hypothesised and this is discussed in some detail.

This book communicates issues of which many practitioners and researchers may not be aware, as publications in this field are often clinically oriented and do not address these fundamental concerns. The absence of a chapter on treatment (other than the one relating to the USA Multimodal Treatment Study of Children with ADHD - the MTA study) is unfortunate, but there are plenty of other sources of information on the subject.

Lindsey Kent Senior Lecturer, University of Birmingham, Division of Neuroscience, Queen Elizabeth Psychiatric Hospital, Mindelsohn Way, Edgbaston, Birmingham BI5 2QZ, UK

\section{Disorders of Body Image}

Edited by David J. Castle \& Katharine

A. Phillips. Petersfield: Wrightson

Biomedical. 2002. 176 pp. $€ 31.00$ (hb).

ISBN I 87I8I6 475

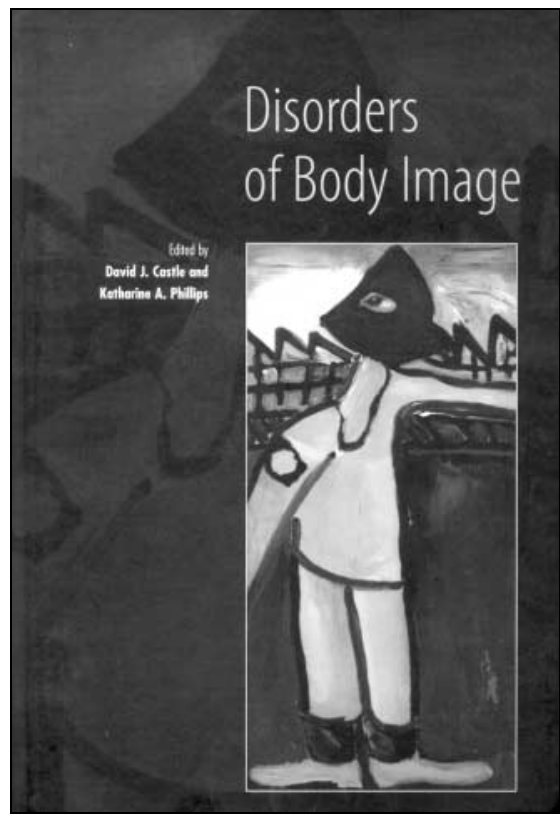

Body image, body image dissatisfaction and body image disturbance can be elusive concepts for us to examine in our patients. Certainly when working with individuals with an eating disorder, suspicions of body image dissatisfaction are often high but it can be difficult to track down. This concise book begins by looking at the neurological basis of body image and goes on to deal with emotional responses to the self. An examination of the ways in which emotion and disgust alter one's perception of oneself is followed by a comprehensive review of socioculturally defined notions of what is and is not acceptable for body size and shape. These different strands give a broad overview of the development of body image in individuals and in cultures. The reader can then start to piece together how this process might go awry in an individual.

The authors focus on a variety of physical and psychiatric conditions in which body image is important and on the varied ways in which clients present to clinicians. The important 'take home' points for me from this book are how difficult body dysmorphic disorder can be to diagnose and how often clinicians miss it. Even when symptoms are florid and obvious, clinicians may not consider the diagnosis and instead identify and treat comorbid conditions such as depression. Clearly many clinicians do not routinely examine body image dissatisfaction and concerns.

There are many demands on our time and many good books that we never get time to read. This volume, however, is small, easy to dip in and out of, and draws together body image and its disorders and treatment in a comprehensive but easy-to-digest format. I would recommend it to any clinician trying to understand the body image disorders of their clients, and to clinicians who never see individuals with a disordered body image, as they may look on their patients with fresh eyes as a result.

Helen Baxter Consultant in Eating Disorders, Nottingham Eating Disorders Service, Nottinghamshire Healthcare NHS Trust, 484 Derby Road, Nottingham NG7 2GT,UK 